\title{
STUDIES ON THE EFFECT OF KINEMATIC VISCOSITY ON ELECTRON-ACOUSTIC CYLINDRICAL AND SPHERICAL SOLITARY WAVES IN A PLASMA WITH TRAPPED ELECTRONS
}

\author{
Subrata Roy ${ }^{1}$, Sandip Saha ${ }^{2}$, Santanu Raut ${ }^{3}$, Apurba Narayan Das ${ }^{4}$ \\ ${ }^{1}$ Department of Mathematics, Cooch Behar Panchanan Barma University, Cooch Behar, 736101, \\ India \\ 2 Department of Mathematics, NIT Silchar, Assam, 788010, India \\ ${ }^{3}$ Department of Mathematics, Mathabhanga College, Coochbehar, 736146, India \\ ${ }^{4}$ Department of Mathematics, Alipurduar Univeristy, Alipurduar, 736121, India \\ send2sroy94@gmail.com,sandip.tfgss@gmail.com,raut_santanu@yahoo.com \\ apuraiganj1964@gmail.com
}

Received: 24 April 2021; Accepted: 26 June 2021

\begin{abstract}
In this article, using the standard reductive perturbation technique (RPT) to the basic governing equations for plasma comprising stationary ions, cold electrons and hot electrons abiding by vortex-like distribution, nonplanar Schamel Burger (NSB) equations is derived. In order to study the propagating properties of Electron acoustic (EA), progressive wave solution is obtained by employing the weighted residual method (WRM). Most of the observations of the EA wave are limited to the plasma environment where the effects of viscosity, collisions, ion streaming velocity are totally neglected. In our present observation, propagation of EA waves in a viscous plasma is described considering a weak damping (by adding a Burgers term) due to the inner particle collision and viscosity. Special attention has been given to study the impact of the other physical parameters in wave propagation in the framework of the Schamel Burgers medium.
\end{abstract}

MSC 2010: 47J35, 70K60, 76Nxx

Keywords: reductive perturbation technique, vortex-like distribution, propagating properties, nonplanar Schamel-Burgers equations, weak damping

\section{Introduction}

Over the last few decades, research works on plasma physics have become highly interesting to the researchers of this field due to their wide applications in many natural phenomena and in different space environments. For the first time, nonlinear phenomena in wave propagation are described using a mechanical analogy in the work of Sagdeev [1]. In 1970, Ikezi et al. [2] initiated an investigation experimentally for observing the behaviors of wave propagation in a plasma environment. Actually, different experimental observations of our universe created a lot of interest in the theory of plasma. In order to understand our early universe [3], we should have 
sufficient knowledge on plasma. Also, from the astronomical observations, it has been confirmed that the plasma extensively exists in active galactic nuclei, the pulsar magnetosphere and the solar atmosphere [4].

In 1995, D'Angelo [5] observed that high-frequency acoustic waves (static particles) and low-frequency dust acoustic waves (mobile dusty particles) can be generated in a plasma medium. Using Kortewegde Vries (KdV) and modified KdV equations, Dubuloz et al. [6] investigated the propagation of solitary waves in both ion acoustic and dust acoustic plasmas using traditional RPT. Subsequently, large numbers of experimental as well as theoretical works have been carried out by several mathematicians and physicists to observe the propagating characteristics of ion acoustic as well as dust ion acoustic waves in plasma system [7-9]. KdV equation and its family are world-renowned because their solutions can explain a number of nonlinear phenomena and structures occurring in different fields of research, for example, fluids, plasma, optics, etc. One of the prominent and most notable waves derived from $\mathrm{KdV}$ is called solitary waves (solitons). Such waves emerge because of the equality between the nonlinearity and the consequence of dispersion. These waves are therefore used to relay information because of their stationary profile after a collision in fiber optic transmissions. In comparison to solitons in energy storage systems, when the soliton is dissipated or damped, all of their characteristics are found to change and decay as time passes $[10,11]$. A large class of theoretical as well as experimental studies have been carried out in different plasma environments, and it is noticed that the evolution of soliton-like solutions in the dissipative systems significantly depend on on certain physical parameters of that medium [12].

In 2011, Pakzad [14] found that there exists a substantial phase shifting in the plasma when the Maxwellian electrons are changed to the non-Maxwellian electron. It is also observed that the phase shift enhances due to the enhance in the ratio of temperatures of hot and cold electron. Moreover, the phase shift is higher in cylindrical EA than in the spherical one. Pakzad showed in [14] that the geometry and nonextensive distribution of hot electrons have changed the width and amplitude of EA in plasma comprising the hot and cold electron population. Recently Demiray et al. [15] had studied on plasma taking the cold electron and hot electron with vortex distribution. Motivated by their works, we intend to find the dissipation effect in the present plasma environment due to the ion viscosity.

However, if the plasma is comprised of stationary ions, cold electrons and hot electrons satisfying trapped or vortex type distribution, then the fractional-order nonplanar KdV equation arises instead of the conventional integer order nonplanar $\mathrm{KdV}$ equation [16]. It is found that the standard techniques described in the references [13] cannot reduce the classical $\mathrm{KdV}$ equation from the fractional one. In this connection, some challenging attempts have been taken by several authors to find a series solution for that type of evolution equation. Thus, due to their large interpretations, many enigmatic phenomena which have not been explained before became one of the main concerns of many mathematicians and physicists, which can be solved by various analytical methods $[17,18]$. In addition, many approximate methods can be employed 
to solve the linear and non-linear differential equations, namely the Adomian decomposition method [19], the residual Galerkin weightage method [20] and the variational iteration method [21]. Dubouloz et al. [6] studied the characteristic of the IAW solitary wave profile in a one dimensional collisionless plasma system composed of stationary ions, electron fluid and Maxwellian hot electrons. But it is assumed that the phase space holes formed because of trapping in a wave potential and thus the distribution cannot be adopted by hot electrons. In general, the hot electrons in space plasmas follow the vortex (trapped) like distribution [15,22] in most of the cases. Recently, a nonplaner EA wave has been studied considering the plasma environment model by neglecting different significant effects such as viscosity, collisions, ion streaming velocity etc $[17,18]$. But some remarkable results given in [23] show that characteristic of wave propagation significantly changed due to the above-mentioned effects. Being aware of the fact that effect of Burgers term in nonplanar Schamel Burgers framework is not considered in earlier works, cylindrical as well as spherical Schamel Burgers equations are derived from the field equation of a dissipative plasma system using RPT. To observe the nonlinear evolution of IAW in this present system, for the first time a new type of progressive wave solution for the NSB equation is explored by employing the Weighted Residual Method.

\section{Mathematical modeling and governing equations}

We assume a homogeneous system with a collisionless plasma comprising of a fluid of cold electrons and hot electrons following distribution of trapped/vortexlike, and stationary ions. The basic one-dimensional governing equations with the aid of the non-dimensionalized variable are structured as follows:

$$
\begin{gathered}
\frac{\partial n}{\partial t}+\nabla \cdot(n v)=0, \\
\frac{\partial v}{\partial t}+(v \cdot \nabla) v=\alpha \nabla \psi+\zeta \nabla^{2} v, \\
\nabla^{2} \psi=\frac{1}{\alpha} n+n_{h}-\left(1+\frac{1}{\alpha}\right) .
\end{gathered}
$$

Where, $n\left(n_{h}\right), v$ and $\psi$ noted as the number of cold (hot) electron density, velocity of cold electron fluid and potential electrostatic wave which are non-dimensionalize by $n_{0}\left(n_{h 0}\right), C_{e}=\left(k_{B} T_{h} / \alpha m\right)^{1 / 2}$ and $k_{B} T_{h}=e$ respectively, with $\alpha=n_{h 0} / n$, where the m, $e$ and $k_{B}$ are noted as electron mass, magnitude of electron charge and the Boltzmann constant. $\beta$ signifies the measure of the inverse temperature of the trapped electron. Moreover, the density of hot electron number, $n_{h}$ (for $\beta<0$ ) is formualized by Schamel as 


$$
n_{h}=I(\psi)+\frac{2}{\sqrt{-\pi \beta}} W_{D}(\sqrt{\beta \psi})
$$

with,

$$
I(x)=[1-\operatorname{erf}(\sqrt{x})] \exp (x)
$$

and,

$$
W_{D}=\exp \left(-x^{2}\right) \int_{0}^{x} \exp \left(y^{2}\right) d y
$$

For more generalizations, see the work of Schamel [24]. For $\psi<1$, the hot electron density can be expressed as follows:

$$
n_{h}=1+\psi-\frac{4}{3 \sqrt{\pi}}(1-\beta) \psi^{3 / 2}+\frac{\psi^{2}}{2}-\frac{8}{15 \sqrt{\pi}}\left(1-\beta^{2}\right) \psi^{5 / 2}+\frac{\psi^{3}}{6}+\cdots
$$

For such plasma, in the present work, we have considered the axially symmetric waves in the case of cylindrical and spherical behavior, which are formualized as:

$$
\begin{gathered}
\frac{\partial n}{\partial t}+\frac{1}{R^{\mu}} \frac{\partial\left(R^{\mu} n v\right)}{\partial R}=0 \\
\frac{\partial v}{\partial t}+v \frac{\partial v}{\partial R}=\alpha \frac{\partial \psi}{\partial R}+\zeta\left[\frac{1}{R^{\mu}} \frac{\partial}{\partial R}\left(R^{\mu} \frac{\partial v}{\partial R}\right)\right] \\
\frac{1}{R^{\mu}} \frac{\partial}{\partial R}\left(R^{\mu} \frac{\partial \psi}{\partial R}\right)=-\frac{1}{\alpha}+\frac{n}{\alpha}+\psi-\frac{4}{3 \sqrt{\pi}}(1-\beta) \psi^{3 / 2}+\cdots
\end{gathered}
$$

where $\mu=1(\mu=2)$ presents the cylindrical (spherical) waves.

Using the reductive perturbation theory, the asymptotic analysis with the aid of equations [8-10] have been done by introducing the slow stretched co-ordinates:

$$
\xi=\varepsilon^{\frac{1}{2}}(R-t), \tau=\varepsilon^{\frac{3}{2}} R,
$$

and the field variables can be written in the form of power series:

$$
\begin{aligned}
& n=1+\varepsilon^{2}\left(n_{1}+\varepsilon n_{2}+\cdots\right) \\
& \psi=\varepsilon^{2}\left(\psi_{1}+\varepsilon \psi_{2}+\varepsilon^{2} \psi_{3}+\cdots\right) \\
& v=\varepsilon^{2}\left(v_{1}+\varepsilon v_{2}+\varepsilon^{2} v_{3}+\cdots\right)
\end{aligned}
$$

We assume a weak damping state in this plasma environment considering ion kinematic viscosity, which leads to

$$
\zeta \approx \varepsilon^{\frac{1}{2}} \zeta_{0}
$$


We substitute the above expansions given by Eqs. (12)-(15) along with stretching coordinates (Eq. (11)) in Eqs. (8)-(10) and considering the coefficients of different power of $\varepsilon$ starting from lower one), we get

$$
\begin{gathered}
n_{1}=v_{1}, \\
\frac{\partial n_{2}}{\partial \xi}-\frac{\partial v_{2}}{\partial \xi}-\frac{\mu v_{1}}{\tau}-\frac{\partial v_{1}}{\partial \tau}=0, \\
v_{1}=-\alpha \psi_{1} \\
\frac{\partial v_{2}}{\partial \xi}+\alpha \frac{\partial \psi_{1}}{\partial \tau}+\alpha \frac{\partial \psi_{2}}{\partial \xi}+\zeta_{0} \frac{\partial^{2} v_{1}}{\partial \xi^{2}}=0, \\
n_{1}=-\alpha \psi_{1}, \\
\frac{\partial^{2} \psi_{1}}{\partial \xi^{2}}-\psi_{2}-\frac{n_{2}}{\alpha}+\frac{4}{3 \sqrt{\pi}}(1-\beta) \psi_{1}^{3 / 2}=0 .
\end{gathered}
$$

Using the above results and writing $\psi_{1}=\phi$, one can easily derive the following cylindrical and spherical Schamel-Burgers equation:

$$
\frac{\partial \phi}{\partial \tau}+a \phi^{\frac{1}{2}} \frac{\partial \phi}{\partial \xi}+b \frac{\partial^{3} \phi}{\partial \xi^{3}}+c \frac{\partial^{2} \phi}{\partial \xi^{2}}+d \phi=0
$$

with $a=\frac{(1-\beta)}{\sqrt{\pi}}, b=\frac{1}{2}, c=-\frac{\zeta_{0}}{2}, d=\frac{\mu}{2 \tau}$.

The extra term $\mu / 2 \tau$ (Eq. (22)), defined as geometrical term in the Eq. (22), arises due to the non-planar geometry and the value of $\mu$ can be 0 (planar), $1 / 2$ (cylindrical), and 1 (spherical waves). When $|\tau| \rightarrow 0$, it is studied that the effect of nonplanar geometry is significant and for higher values of $|\tau|$, it becomes an weak formation.

\section{Analytical approximate solution for nonplanar Schamel-Burgers equation}

Generally, one cannot solve the Eq. (22) for an exact analytical solution and one has to attempt to get its analytical approximate solution. For sufficiently large values of $\tau$ and $\zeta_{0}=0$, the nonplanar Schamel-Burger Eq. (22) may be rewritten as 


$$
\frac{\partial \phi}{\partial \tau}+a \sqrt{\phi} \frac{\partial \phi}{\partial \xi}+b \frac{\partial^{3} \phi}{\partial \xi^{3}}=0
$$

we assume the solitary wave solution of the Eq. (23) as

$$
\phi=\lambda_{0} \operatorname{sech}^{4} \eta_{0}, \quad \eta_{0}=w\left(\xi-V_{0} \tau\right)
$$

with

$$
w^{2}=\frac{a \lambda_{0}^{1 / 2}}{30 b}, \quad V_{0}=\frac{8 a \lambda_{0}^{1 / 2}}{15}
$$

where $\lambda_{0}$ is the constant amplitude. The form of the solution given by Eq. (24) motivated us to propose a progressive wave solution for the evolution Eq. (22) as

$$
\phi=\lambda(\tau) \operatorname{sech}^{4} \eta, \quad \eta=w(\tau)(\xi-V(\tau))
$$

with

$$
w^{2}(\tau)=\frac{a \lambda(\tau)^{1 / 2}}{30 b}, V^{\prime}(\tau)=\frac{8 a \lambda(\tau)^{1 / 2}}{15}
$$

where $V^{\prime}(\tau)$ denotes differentiation of the function $V(\tau)$ w.r.t $\tau$. As a matter of fact, the solutions given in Eq. (26) and Eq. (27) are formally the same with those given in Eq. (24) and Eq. (25) except that in Eq. (27), $\lambda(\tau)$ is to be determined. Using the results Eq. (26), Eq. (27) in the Eq. (22), we note that the equation is not satisfied identically; there will be rather a residue term $R(\eta, \tau)$ given by

$$
R(\eta, \tau)=\left[\lambda^{\prime}+16 c \lambda w^{2}+d \lambda-\frac{4 \lambda w^{\prime} \eta}{w} \tanh ^{2}\right] \operatorname{sech}^{4} \eta-20 \operatorname{cw}^{2} \lambda \operatorname{sech}^{6} \eta
$$

The above expression cannot be made zero point by point. Here, we note that the residue term $R(\eta, \tau)$ is an even function of the variable $\eta$. Using WRM [17, 18], we obtain a differential equation for $\lambda(\tau)$. To get a strong restrictions on the coefficient $\lambda(\tau)$, we choose $\operatorname{sech}^{4} \eta$ as a weighting function. Multiplying the result of Eq. (28) by the weighting function and integrating with limit $-\infty$ to $\infty$ for $\eta$ and setting the result equal to zero, we get

$$
\lambda^{\prime}+16 c \lambda w^{2}+d \lambda-\frac{\lambda w^{\prime}}{2 w}-\frac{160 c w^{2} \lambda}{9}=0
$$

removing $w$ from Eq. (29) with the aid of Eq. (27) one gets

$$
\lambda^{\prime}+\frac{4 \mu}{7 \tau} \lambda=\frac{64 a c}{945 b} \lambda^{\frac{3}{2}}
$$


From this, one can easily obtain

$$
\lambda(\tau)=\left(P \tau^{\frac{2 \mu}{7}}+\frac{32 a c \tau}{\left(\frac{2 \mu}{7}-1\right) 945 b}\right)^{-2}
$$

where $P$ is the constant of integration. Using $\lim _{\tau \rightarrow \tau_{0}} \lambda(\tau)=\lambda_{0}$, the wave amplitude defined earlier, we see that the result (Eq. (29)) becomes

$$
\lambda(\tau)=\lambda_{0}\left(\frac{\tau_{0}}{\tau}\right)^{\frac{4 \mu}{7}}\left(1+\frac{32 a c \lambda_{0}^{\frac{1}{2}} \tau_{0}}{\left(\frac{2 \mu}{7}-1\right) 945 b}\left[\left(\frac{\tau_{0}}{\tau}\right)^{\frac{2 \mu}{7}-1}-1\right]\right)^{-2}
$$

Using Eq. (32) in the expression of Eq. (27) and integrating, we get

$$
V(\tau)=\frac{8 a c \tau_{0} \lambda_{0}^{\frac{1}{2}}}{15}-\frac{63}{4 c} \ln \left(1+\frac{32 a c \lambda_{0}^{\frac{1}{2}} \tau_{0}}{\left(\frac{2 \mu}{7}-1\right) 945 b}\left[\left(\frac{\tau_{0}}{\tau}\right)^{\frac{2 \mu}{7}-1}-1\right]\right)
$$

where we have set $V\left(\tau_{0}\right)=\frac{8 a c \tau_{0} \lambda_{0}^{\frac{1}{2}}}{15}$. Equation (27) gives

$$
w(\tau)=\sqrt{\frac{a \lambda_{0}^{\frac{1}{2}}\left(\frac{\tau_{0}}{\tau}\right)^{\frac{2 \mu}{7}}\left(1+\frac{32 a c \lambda_{0}^{\frac{1}{2}} \tau_{0}}{\left(\frac{2 \mu}{7}-1\right) 945 b}\left[\left(\frac{\tau_{0}}{\tau}\right)^{\frac{2 \mu}{7}-1}-1\right]\right)^{-1}}{30 b}}
$$

Thus, the final solution may be expressed by

$$
\phi(\xi, \tau)=\lambda(\tau) \operatorname{sech}^{4}[w(\tau)(\xi-V(\tau))]
$$

where $\tau>>\tau_{0}$ and $\lambda_{0}$ is the initial amplitude and $\lambda(\tau), V(\tau)$, and $w(\tau)$ are given by Equations (32)-(34).

\section{Results and discussions}

The analytical solution given by Eq. (35) of the modified cylindrical (spherical) $\mathrm{KdV}$ equation in the presence of the kinematic viscosity coefficient has been used to study the physical behavior of model parameters and three different observations have been depicted in Figures 1-4: In this section the propagating behaviors of cylindrical and spherical IAW for the variations in different parameters, viz., $\tau, \beta, \zeta_{0}$, and $\mu$ are analyzed from a numerical stand point in Figures 1-4. In Figure 1a and $1 b$, the analytical solutions given by Eq. (35) of Eq. (22) have been presented in cylindrical and spherical geometry respectively. In both cases, the amplitude of the solitons decrease with the increase in time. In Figure 1, the evolution of a solitary structure is shown in planer $(\mu=0)$ and nonplaner $(\mu=1$ and $\mu=2)$ geometry for $\beta=-1$, $\lambda_{0}=0.8, \tau=10, \zeta_{0}=0.2, \tau_{0}=4$. It is found that a nonplaner soliton moves with 


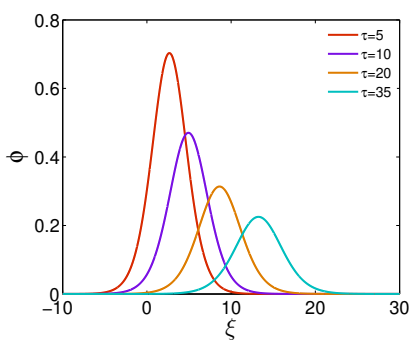

(a)

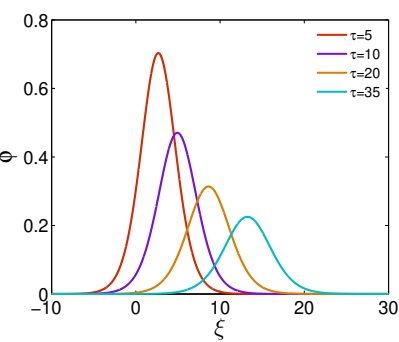

(b)

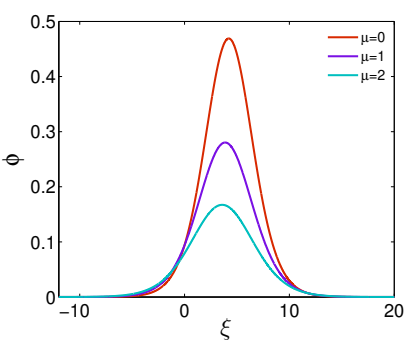

(c)

Fig. 1. Profiles of $\phi(\xi, \tau)$ given by Eq. (35): a) when $\beta=-1, \mu=1, \lambda_{0}=0.8, \zeta_{0}=0.2, \tau_{0}=4$, b) when $\beta=-1, \mu=2, \lambda_{0}=0.8, \zeta_{0}=0.2, \tau_{0}=4$, c) when $\beta=-1, \lambda_{0}=0.8$, $\tau=10, \zeta_{0}=0.2, \tau_{0}=4$

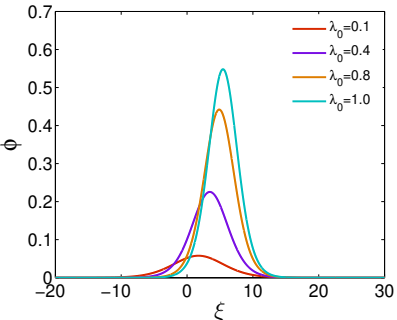

(a)

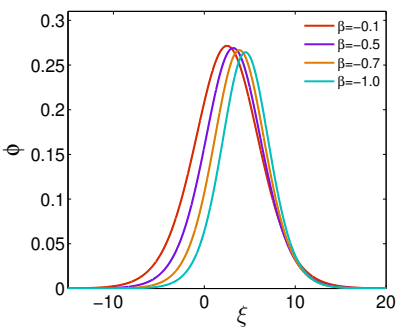

(d)

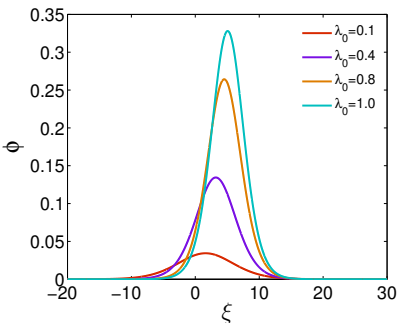

(b)

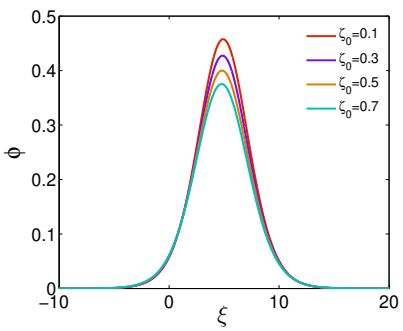

(e)

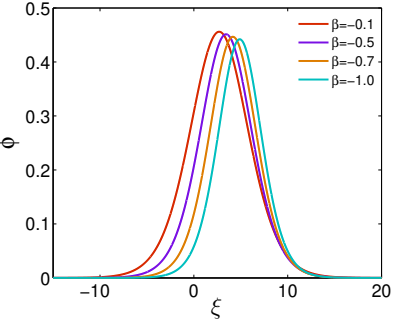

(c)

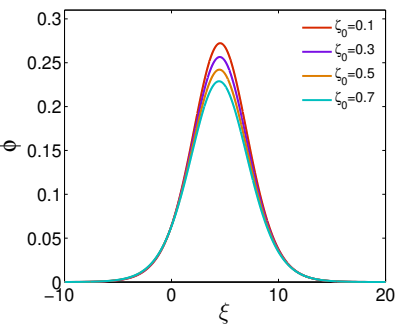

(f)

Fig. 2. Profiles of $\phi(\xi)$ given by Eq. (35): a) when $\beta=-1, \zeta_{0}=0.2, \mu=1, \tau_{0}=4, \tau=10$, b) when $\beta=-1, \zeta_{0}=0.2, \mu=2, \tau_{0}=4, \tau=10$, c) when $\mu=1, \lambda_{0}=0.5, \tau=10$, $\zeta_{0}=0.2, \tau_{0}=4$, d) $\mu=2, \tau_{0}=4, \tau=10$, e) when $\beta=-1, \mu=1, \tau_{0}=4, \beta=-1$, $\tau=10$,f) when $\beta=-1, \mu=2, \tau_{0}=4, \beta=-1, \tau=10$

higher amplitude compared to the planer one. During the evolution, it is also observed from Figure 1c that the amplitude of the cylindrical soliton is higher than that of the planer one however, it is always less than that of the spherical soliton like solution. Figure $2 \mathrm{a}$ and $2 \mathrm{~b}$ are drawn to present the variation of the amplitude of the soliton in cylindrical and spherical geometry respectively due to the change in the parameter $\lambda_{0}$ of the soliton. It is noticed that higher $\lambda_{0}$ leads to form the soliton with higher amplitude however, an increase in $\lambda_{0}$ makes the soliton narrower. Figure $2 \mathrm{c}$ and $2 \mathrm{~d}$ present 


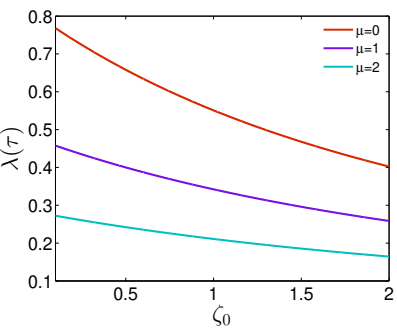

(a)

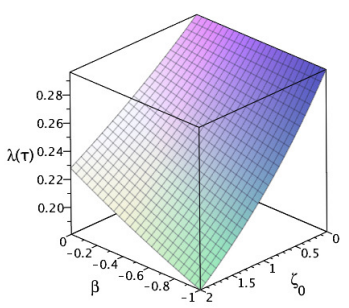

(d)

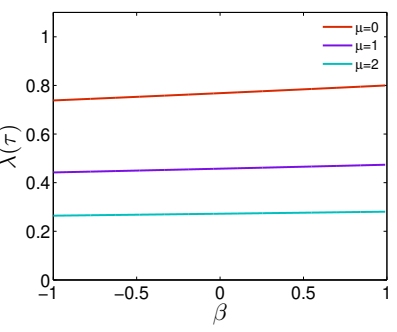

(b)

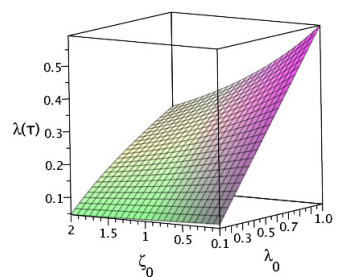

(e)

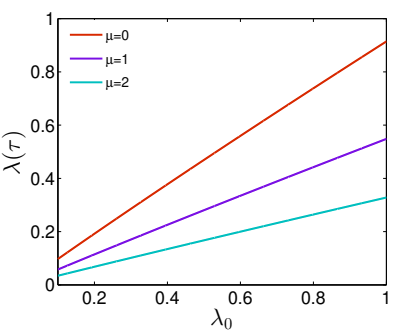

(c)

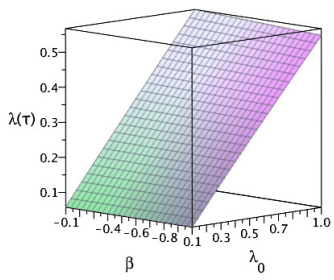

(f)

Fig. 3. Variation of Amplitude $\lambda$ given by Eq. (32): a) for $\beta=-1, \lambda_{0}=0.8, \tau_{0}=4, \tau=10$, b) when $\lambda_{0}=0.8, \zeta_{0}=0.2, \tau=10, \tau_{0}=4$, c) when $\beta=-1, \zeta_{0}=0.2, \tau_{0}=4$, $\tau=10$. Variation of Amplitude Eq. (32) in 3d view, d) for $\lambda_{0}=0.8, \tau_{0}=4$, $\tau=10, \mathrm{e})$ for $\beta=-1, \tau_{0}=4, \tau=10, \mathrm{f}$ ) for $\zeta_{0}=0.2, \tau_{0}=4, \tau=10$

the variations in wave propagation with the variations in $\beta$ for cylindrical and spherical geometrical space respectively. As $\beta$ decreases, a larger portion of the electron becomes free from trapping and naturally magnitude of the electric field diminishes which causes loss in potential energy of the system. Thus in both the cases (cylindrical and spherical), we observed that the soliton becomes small in size (that is, the soliton with lower amplitude as well as lower width). The most remarkable result in this investigation follows from the Figure 2e and 2f. The impact of the Burgers term in wave propagation in cylindrical and spherical geometry is shown in Figure 2e and $2 \mathrm{f}$ where an enhancement in the Burger term causes the soliton to go dipper. This type of nonlinear characteristic in solitary structure is expected as enhancement in Burgers effect leads to increase dissipation in the system. Some $2 D$ and $3 D$ figures are drawn in Figures 3a-f for a clear presentation of the variation in amplitude due to the variation in different physical parameters. In Figure 3a, we see the reduction of the affects in the amplitude for choosing nonplanar geometrical space as a field media. Moreover slow diminishing trend in the amplitude of the solitons appear due to the increase in $\zeta_{0}$. Figure $3 \mathrm{~b}$ shows that no significant change in amplitude is found due to an increase in the distribution parameter $\beta$. It is confirmed from Figure $3 \mathrm{c}$ that the amplitude of the soliton substantially increases with the increase in $\lambda_{0}$. Figures $3 \mathrm{~d}-\mathrm{f}$ exhibit the variation in amplitude in different parametric plane. Nonlinear evolution of the soliton like the solution in cylindrical and spherical geometrical space are presented in Figures 4a-b, in which singularity of the solution is confirmed at $\tau=0$. 


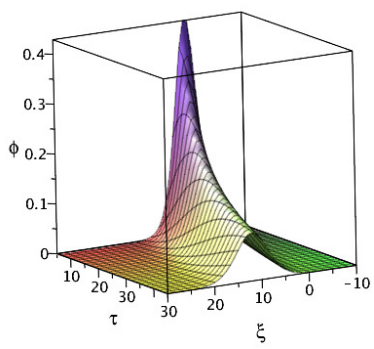

(a)

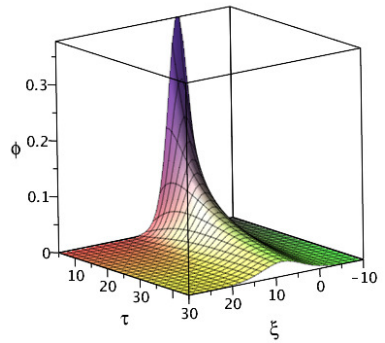

(b)

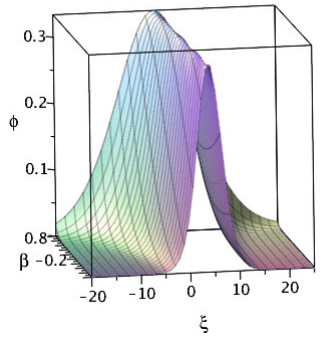

(c)

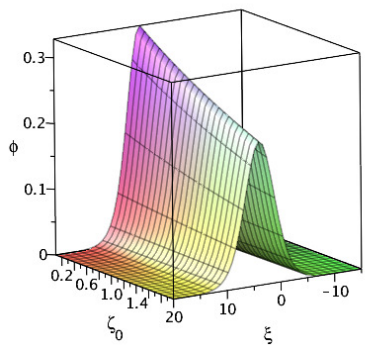

(d)

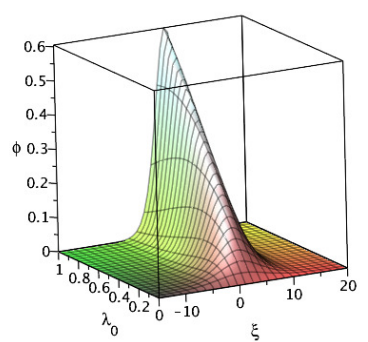

(e)

Fig. 4. 3d Profiles of for Solution Eq. (35): a) for $\mu=1, \lambda_{0}=0.8, \tau_{0}=4, \zeta_{0}=0.2, \beta=-1$, b) for $\mu=2, \lambda_{0}=0.8, \tau_{0}=4, \beta=-1$. c) when $\mu=1, \lambda_{0}=0.8, \tau_{0}=4, \zeta_{0}=0.2, \tau=10$,

d) when $\mu=1, \lambda_{0}=0.8, \tau_{0}=4, \beta=-1, \tau=10$, e) when $\tau_{0}=4, \mu=1, \beta=-1, \tau=10$

Figure $4 \mathrm{c}$ shows how the change in the parameter $\beta$ affect the width of the soliton. A strong enhancing trend in width is found in Figure $4 \mathrm{c}$ due to the decrease in the parameter $\beta$. Figure $4 \mathrm{~d}$ and $4 \mathrm{e}$ show that the significant effect of the parameters $\zeta_{0}$ and $\lambda_{0}$ on amplitude of the soliton.

\section{Conclusion}

In this literature, the propagating behaviors of the EA wave in cylindrically and spherically symmetric plasmas comprising hot electrons with vortex-like distribution have been studied in the framework of the nonplanar Schamel-Burgers model. To our best knowledge, for the first time, the dissipation effect is considered in the nonplanar Schamel model, and their significant effects have been noticed in wave propagation. The entire investigation has been carried out to find the effect of other physical parameters in the Schamel Burgers medium, and the major observations can be summarized as follows:

During the investigation, it has been observed from the figures illustrated with the variations in parameters that the spherical soliton is always somewhat bigger than 
the cylindrical one due to the presence of the modification term $(\mu / 2 \tau)$ in the NSB equation.

The values of wave velocity and wave amplitude increase with the increase in the value of positive ion constant $\lambda_{0}$. It is also found that the width of the wave decreases as the value of $\lambda_{0}$ increases. Moreover, the component value of the expression $\left(\frac{\tau_{0}}{\tau}\right)^{\frac{2 \mu}{7}-1}$ decreases with the increase in the value of $\tau$ and as a result, the value of the wave velocity and the value of the expression, $\left(\frac{\tau_{0}}{\tau}\right)^{\frac{2 \mu}{7}-1}$ also decrease.

A significant effect in wave propagation has been observed due to the variation in $\beta$. The decrease in $\beta$ releases the free electron from trapping and thus the totality of magnitude of the electric field is lost. As a result, the soliton becomes small in size and a left-hand shifting in solitary motion is also found. As expected, a diminishing trend in the amplitude of the soliton appears for enhancing $\zeta_{0}$ which causes an increase in the dissipation effect in the system.

\section{Acknowledgement}

The paper has been revised in the light of reviewer's valuable suggestions. Thanks are due to the reviewers for their comments on the earlier version of this presentation. Financial support from UGC [No. 1106/2018], INDIA to carry out this research work is also thankfully acknowledged by the first author.

\section{References}

[1] Sagdeev, Z.R. (1966). Reviews of Plasma Physics. vol 4 ed New York: Consultants Bureau.

[2] Ikezi, H., Taylor, R., \& Baker, D. (1970). Formation and interaction of ion-acoustic solitions. Phys. Rev. Lett., 44 (11)

[3] Weinberg, S. (1972). Gravitation and Cosmology. New York: Wiley.

[4] Miller, R.H., \& Witter, J.P. (1987). Active Galactic Nuclei, 202.

[5] D'Angelo, N. (1995). Coulomb solids and low-frequency fluctuations in RF dusty plasmas. J. Phys. D, 28, 1009

[6] Dubouloz, N., Pottelette, R., Malingre, M., \& Treumann, A.R. (1991). Generation of broadband electrostatic noise by electron acoustic solitons. Geophysical Research Letters, 18(2), 155-158.

[7] El-Monier, Y.S., \& Atteya, A. (2020). Dynamics of ion-acoustic waves in nonrelativistic magnetized multi-ion quantum plasma: the role of trapped electrons. Waves in Random and Complex Media, 1-19.

[8] Gill, S.T., \& Bansal, S. (2021). Collisionless damping of nonplanar dust acoustic waves due to dust charge fluctuation in nonextensive polarized plasma. Physica Scripta, 96(7), 075605.

[9] Tolba, E.R. (2021). Propagation of dust-acoustic nonlinear waves in a superthermal collisional magnetized dusty plasma. The European Physical Journal Plus, 136(1), 1-15.

[10] Mandi, L., Mondal, K.K., \& Chatterjee, P. (2019). Analytical solitary wave solution of the dust ion acoustic waves for the damped forced modified Korteweg-de Vries equation in q-nonextensive plasmas. The European Physical Journal Special Topics, 228(12), 2753-2768. 
[11] Mondal, K.K., Roy, A., Chatterjee, P., \& Raut, S. (2020). Propagation of ion-acoustic solitary waves for damped forced Zakharov Kuznetsov equation in a relativistic rotating magnetized electron-positron-ion plasma. International Journal of Applied and Computational Mathematics, 6(3), 1-17.

[12] Raut, S., Mondal, K.K., Chatterjee, P., \& Roy, A. (2021). Propagation of dust-ion-acoustic solitary waves for damped modified Kadomtsev-Petviashvili-Burgers equation in dusty plasma with a q-nonextensive nonthermal electron velocity distribution. SeMA, 1-23.

[13] Nishida, Y., Nagasawa, T., \& Kawamata, S. (1978). Experimental verification of the characteristics of ion-acoustic cylindrical solitons. Phys. Lett., 69, 196-198.

[14] Pakzad, R.H. (2011). Ion acoustic shock waves in dissipative plasma with superthermal electrons and positrons. Astrophysics and Space Science, 331(1), 169-174.

[15] Demiray, H., \& El-Zahar, R.E. (2018). Cylindrical and spherical solitary waves in an electronacoustic plasma with vortex electron distribution. Physics of Plasmas, 25(4), 10.1063/1.5021729.

[16] Demiray, H., \& Bayindir, C. (2015). A note on the cylindrical solitary waves in an electronacoustic plasma with vortex electron distribution. Physics of Plasmas, 22(9), 092105.

[17] Demiray, H. (2020). Analytical solution for nonplanar waves in a plasma with q-nonextensive nonthermal velocity distribution: Weighted residual method. Chaos, Solitons \& Fractals, 130, 109448.

[18] Demiray, H., El-Zahar, R.E., \& Shan, S.A. (2020). On progressive wave solution for non-planar KDV equation in a plasma with q-nonextensive electrons and two oppositely charged ions. TWMS Journal of Applied and Engineering Mathematics, 10(2), 532-546.

[19] Rach, R., Wazwaz, M.A., \& Duan, S.J. (2013). A reliable modification of the Adomian decomposition method for higher-order nonlinear differential equations. Kybernetes, 42(2), 282-308.

[20] Shukla, K.P. (2001). A survey of dusty plasma physics. Phys. Plasmas, 8, 1791-1803.

[21] Ebaid, A., Rach, R., \& El-Zahar, E. (2017). A new analytical solution of the hyperbolic Kepler equation using the Adomian decomposition method. Acta Astronautica, 138, 1-9.

[22] Paul, A., Mandal, G., Amin, R.M., \& Chatterjee, P. (2020). Analysis of solution of damped modified-KdV equation on dust-ion-acoustic wave in presence of superthermal electrons. Plasma Physics Reports, 46(1), 83-89.

[23] Mamun, A.A., \& Shukla, K.P. (2002). Electrostatic solitary and shock structures in dusty plasmas. Physica Scripta, 98, 107.

[24] Schamel, H. (1972). Stationary solitary, snoidal and sinusoidal ion acoustic waves. Plasma Physics, 14(10), 905. 